\title{
SHUTTLE-RUN INTERVAL TRAINING WITH MORE DIRECTIONAL CHANGES INDUCES SUPERIOR GAINS IN SHUTTLE SPRINT PERFORMANCE IN FEMALE PROFESSIONAL FUTSAL PLAYERS
}

original paper

(1) University School of Physical Education in Wroclaw

DOI: https://doi.org/10.5114/hm.2018.79623

\author{
ANDERSON SANTIAGO TEIXEIRA ${ }^{1}$, FRANCIMARA BUDAL ARINS ${ }^{1}$, \\ RICARDO DANTAS DE LUCAS ${ }^{1}$, LORIVAL JOSE CARMINATTI ${ }^{2}$, \\ NAIANDRA DITTRICH ${ }^{1}$, FABIO YUZO NAKAMURA ${ }^{3,4}$, IRINEU LOTURCO ${ }^{5}$, \\ LUIZ GUILHERME ANTONACCI GUGLIELMO ${ }^{1}$ \\ ${ }^{1}$ School of Sports, Federal University of Santa Catarina, Florianópolis, Brazil \\ ${ }^{2}$ College of Health and Sport Science, Santa Catarina State University, Florianópolis, Brazil \\ ${ }^{3}$ Department of Medicine and Aging Sciences, Gabriele d'Annunzio University, Chieti-Pescara, Italy \\ ${ }^{4}$ College of Healthcare Sciences, James Cook University, Queensland, Australia \\ ${ }^{5}$ Nucleus of High Performance in Sport, São Paulo, Brazil
}

\section{ABSTRACT}

Purpose. To compare the chronic (5-week) effects of two shuttle run interval training modes with one (shuttle running interval training 1, SRIT-1) and three (SRIT-3) directional changes on blood lactate responses ([La]), strength, power, and speed capacities of female futsal players.

Methods. Fourteen players performed unloaded squat (SJ) and countermovement (CMJ) jumps. Isokinetic assessments of lower limbs and a single 40-m shuttle-sprint test took place before and after the training program. [La] was measured after each running set during the $1^{\text {st }}$ and $10^{\text {th }}$ training session.

Results. [La] values were very likely reduced in the $10^{\text {th }}$ compared with the $1^{\text {st }}$ session in both groups. After SRIT-3, very likely moderate improvements occurred in the $40-\mathrm{m}$ shuttle-sprint speed test ( $\Delta=+2.9 \%$; $90 \%$ CI: $1.7-4.1$ ), while changes were unclear following SRIT-1 (1.0\%; from -1.0 to 3.1). Changes in SJ (SRIT-1: +7.8\%; 0.8-15.3; SRIT-3: +9.2\%; 4.4-14.2) and CMJ height (SRIT-1: +7.0\%; 1.1-13.1; SRIT-3: +8.4\%; 3.7-13.3) were likely to very likely beneficial following both training protocols. Knee extensor concentric peak torque was likely and very likely increased in the post-training period for both SRIT-1 (+18.3\%; 1.9-37.4) and SRIT-3 (+17.3\%; 4.6-31.6), respectively. Knee extensor eccentric peak torque was very likely improved after SRIT-3 (+9.5\%; 4.7-14.4), but unclear following SRIT-1 (+3.4\%; from -6.3 to 14.1). Following SRIT-3, changes in the 40-m shuttle-sprint speed were likely (+1.8\%; from -0.4 to 4.1$)$ superior to those observed in SRIT-1.

Conclusions. SRIT-3, probably because of the higher number of horizontal accelerations, might be more effective than SRIT-1 (with fewer changes of direction) to induce greater adaptations in 40-m shuttle sprint in female futsal players.

Key words: indoor soccer, neuromuscular adaptation, sprinting performance, blood lactate responses, intermittent training

\section{Introduction}

Futsal is a multiple-sprint sport played on a hard court surface $(40 \times 20 \mathrm{~m})$ between two teams (5-a-side) in two halves of 20 minutes separated by a halftime interval of 10 minutes [1]. This sport discipline involves a complex range of high-intensity locomotor activities (e.g., accelerations, decelerations, dribbles, jumps, rapid change of directions, and side-cutting movements) and is characterized as a physically demanding modality, requiring both aerobic and anaerobic fitness to cope with the multiple requirements of the game [2-4]. Futsal players cover a total distance of 3-4 km during an official match [5]. Sprinting and high-intensity running activities account for $5 \%$ and $12 \%$ of the total playing time, respectively [3]. During an official match,

Correspondence address: Anderson Santiago Teixeira, Physical Effort Laboratory, School of Sports, Federal University of Santa Catarina, Florianópolis - SC, Brazil, e-mail: anderson.santeixeira@gmail.com

Received: September 11, 2018

Accepted for publication: November 12, 2018

Citation: Teixeira AS, Arins FB, De Lucas RD, Carminatti LJ, Dittrich N, Nakamura FY, Loturco I, Guglielmo LGA. Shuttle-run interval training with more directional changes induces superior gains in shuttle sprint performance in female professional futsal players. Hum Mov. 2018;19(5)special/issue:40-51; doi: https://doi.org/10.5114/hm.2018.79623. 
players can perform up to $26 \pm 13.3$ sprints with a recovery time between them of $59.3 \pm 66.1 \mathrm{~s}$, and a mean sprint bout distance of $13.7 \pm 6.1 \mathrm{~m}$ [2]. No decrement of sprint performance was found between the halves; the unlimited substitutions allowed by futsal rules are a possible explanation for this outcome [2]. Owing to these high demands and the accumulation of fatigue during matches and training sessions, coaches and technical staff need to plan activities that allow an integral development of the player's physical and physiological traits during short periods of time (4-6 weeks), such as the preseason phases [6, 7].

In addition to aerobic fitness features [7, 8], in view of the high neuromuscular demands in modern futsal [9], strength and conditioning specialists should also properly optimize the athletes' speed and power capacities [10]. From a practical perspective, with the consideration of the 'interference phenomenon,' commonly reported in team sports [11, 12], it does not seem straightforward to improve neuromechanical capabilities throughout preseason phases, where futsal players are frequently exposed to high volumes (ca. $52-88 \%$ of the total time) of predominantly aerobic training activities (i.e., technical and tactical sessions) [13]. Briefly, the interference phenomenon can be understood as a multifactorial process that refers to the concurrency that occurs between aerobic and strength-power training effects on neuromuscular adaptations [11]. For instance, in an earlier work, Nakamura et al. [9] demonstrated that male futsal players had their neuromuscular performance (i.e., sprinting speed and vertical jump ability) decreased after a short aerobic-oriented preseason phase.

However, it seems that the impact of the concurrent training, also defined as the simultaneous integration of resistance and endurance exercise into a training program, on neuromuscular performance [14] can be reduced through the use of some specific strategies. As an alternative to plyometrics or loaded squat jump (SJ) exercises [10, 12, 15], it has been shown that accelerations performed during shuttle running at submaximal intensities may impose higher physiological and mechanical demands on working muscles [16], emerging as a potential training method to counteract the interference effect on neuromuscular adaptations during the preseason phases.

Originally, the shuttle running interval training (SRIT) model requires successive changes of direction, accelerations, and decelerations (thus increasing neuromuscular demand) during intermittent running bouts performed at intensities greater (105-110\%) than maximal aerobic speed in team sports players (e.g., soccer and handball) $[17,18]$. The configuration of this training scheme is determined by the organization and manipulation of different variables, such as intensity, number of sets, duration of sets, time between the sets, and intensity of recovery between the sets [19]. Along with these variables, the total number of changes of direction seems to be a key parameter for designing optimal SRIT strategies. Indeed, Akenhead et al. [16] have already indicated that the inclusion of more changes of direction (9 vs. 19 vs. 29 turns in a total course of $600 \mathrm{~m}$ ) during submaximal shuttle running speeds ( 2.50 vs. 3.25 vs. $4.00 \mathrm{~m} \cdot \mathrm{s}^{-1}$ ) is an efficient way to increase the percentage of time spent accelerating per turn in professional footballers. Additionally, it was recently shown that high-intensity running training with more directional changes can be a more effective training strategy to induce superior gains in athletic performance in female basketball players [20].

On the basis of these factors, the SRIT model could be another feasible approach to simultaneously develop intermittent endurance, speed, and power performance in team sport athletes, especially in female players, who usually present lower levels of aerobic fitness, strength, and power than their male peers [15, 21]. Within a mechanical perspective, SRIT schemes involving distinct amounts of changes of direction per a running bout can significantly influence the time that a given athlete spends accelerating and, consequently, the time spent applying horizontal force against the ground (which seems to be crucial for enhancing acceleration capabilities in team sport athletes) [22]. In addition, since each change of direction requires a braking force (high eccentric activity) followed by a horizontal propulsive force, these training types (i.e., SRIT-1 and SRIT-3) can also elicit a high blood lactate production [16], thus increasing the physiological/metabolic load placed on a player. Assessments of blood lactate concentration ([La]) responses during submaximal conditions can be used to monitor training-induced changes without requiring maximal efforts of the players [23]. Indeed, a lower [La] at the same submaximal exercise intensity after a training period indicates an important muscle peripheral adaptation, signalling meaningful changes in muscle metabolism [23]. However, particularly in futsal, the effects induced by different SRIT models on [La], strength, power, and speed qualities in female futsal players have not been extensively addressed yet. Indeed, the majority of data describing training-induced changes are limited to male futsal players [6, 7, 13, 24].

Therefore, the present study aimed to compare the effects of two SRIT models, with one (SRIT-1) and three 
(SRIT-3) directional changes per a running bout, implemented over a period of 5 weeks, on [La], strength, power, and speed capacities of female futsal players. Our hypothesis was that both the SRIT-1 and SRIT-3 models would induce meaningful improvements on endurance capacity, strength (assessed by means of isokinetic tests), and jump performance. However, because of the higher number of horizontal accelerations in SRIT-3, we hypothesized that this training model would have a superior effect on the sprinting speed when compared with SRIT-1.

\section{Material and methods}

\section{Participants}

Fourteen young female outfield futsal players (age: $18.71 \pm 1.94$ years; height: $160.57 \pm 3.86 \mathrm{~cm}$; body mass: $59.23 \pm 8.00 \mathrm{~kg}$; \% body fat: $18.89 \pm 4.85 \%$; systematic futsal practice: $4.64 \pm 1.98$ years) belonging to the same professional team competing in the Brazilian National Division League were originally recruited to participate in this study. At the time of study, all the players had had at least 3 years of experience $(4.5 \pm$ 1.2 years) in futsal practice and trained 6 days per week, with 1 or 2 daily training sessions. The inclusion criteria for the study were: (1) regular participation in $100 \%$ of training sessions during the period of investigation; (2) not suffering from injuries during the same period; and (3) having completed all physical fitness assessments. Participation was voluntary and the players could withdraw at any time.

\section{Experimental design}

A parallel two-group, matched-work, longitudinal experimental design was used to assess the changes in futsal-related physical fitness attributes induced by two different SRIT protocols. The whole study was conducted within 7 weeks, which included a 5-week training period. Training was implemented during the preseason phase (from the beginning of March to the end of April) in addition to a regular futsal training schedule proposed by coaches and trainers, and included: (1) pre-training testing $\left(1^{\text {st }}\right.$ week); (2) a 5-week intervention training period (from $2^{\text {nd }}$ to $6^{\text {th }}$ week); and (3) post-training testing ( $7^{\text {th }}$ week). Owing to the game schedule (in-season period) that began in May, the intervention period lasted only 5 weeks. To ensure that both groups presented similar pre-training average values for each performance variable, the players were pair-matched on the basis of their baseline performance in the Futsal Intermittent Endurance Test (FIET) and randomly assigned to either the SRIT-1 $(n=7)$ or SRIT-3 $(n=7)$ training group. The group allocation was performed by a computer-generated random sequence created by a researcher, blinded on the participants' identities.

\section{Procedures}

\section{Training intervention}

During the intervention period, two different training regimens were implemented based on the peak speed (PS) derived from FIET ( PS $_{\text {FIET }}$ ), being both training regimens performed by athletes twice a week (every Tuesday and Thursday). The training protocols consisted of shuttle-run intervals organized in 4 sets of 4-min bouts with 3-min rest intervals between the sets (Figure 1). The main difference between the two training protocols was the duration of the exercise and rest, with one comprising $7.5 \mathrm{~s}$ running and $7.5 \mathrm{~s}$ pause (SRIT-1), and the other $15 \mathrm{~s}$ running and $15 \mathrm{~s}$ pause (SRIT-3) per bout. In the SRIT-1 and SRIT-3 models, the athletes performed during each 4-min bout, the total of $16 \times 7.5 \mathrm{~s}$ and $8 \times 15 \mathrm{~s}$ shuttle-runs (with a change of direction every $3.75 \mathrm{~s}$ in each model), respectively. The intensity used for the SRIT-1 and SRIT-3 models was $89 \%$ and $86 \%$ of $\mathrm{PS}_{\text {FIET, }}$, respectively [25]. This difference was set to account for the time necessary to perform each change of direction [18], which occurred 3 times more in SRIT-3 compared with SRIT-1. After the 5-week training period, players who trained in SRIT-1 and SRIT-3 models should have completed the total of 640 and 960 turns, respectively. The average running pace performed by the athletes between the start and return lines for each training protocol was dictated by a prerecorded audio cue, emitting beeps every $3.75 \mathrm{~s}$. The distance covered by each athlete during the training sessions was individualized in accordance with their respective PS $_{\text {FIET }}$. Heart rate (HR) was monitored during all training sessions with a commercially available telemetry system (Polar S610; Polar Electro Oy). Throughout the training period, the external load (i.e., running intensity) was increased when the athletes had HR mean values lower than 90\% of the individual maximal HR (HRmax) for two consecutive training sessions. In that situation, the distance was increased by $1 \mathrm{~m}$, which corresponds to a $0.9 \mathrm{~km} / \mathrm{h}$ increment. During the $10^{\text {th }}$ training session, the external load (i.e., running intensity in the FIET) applied was the same as the one recorded in the $1^{\text {st }}$ training session, in order to analyse training-induced 

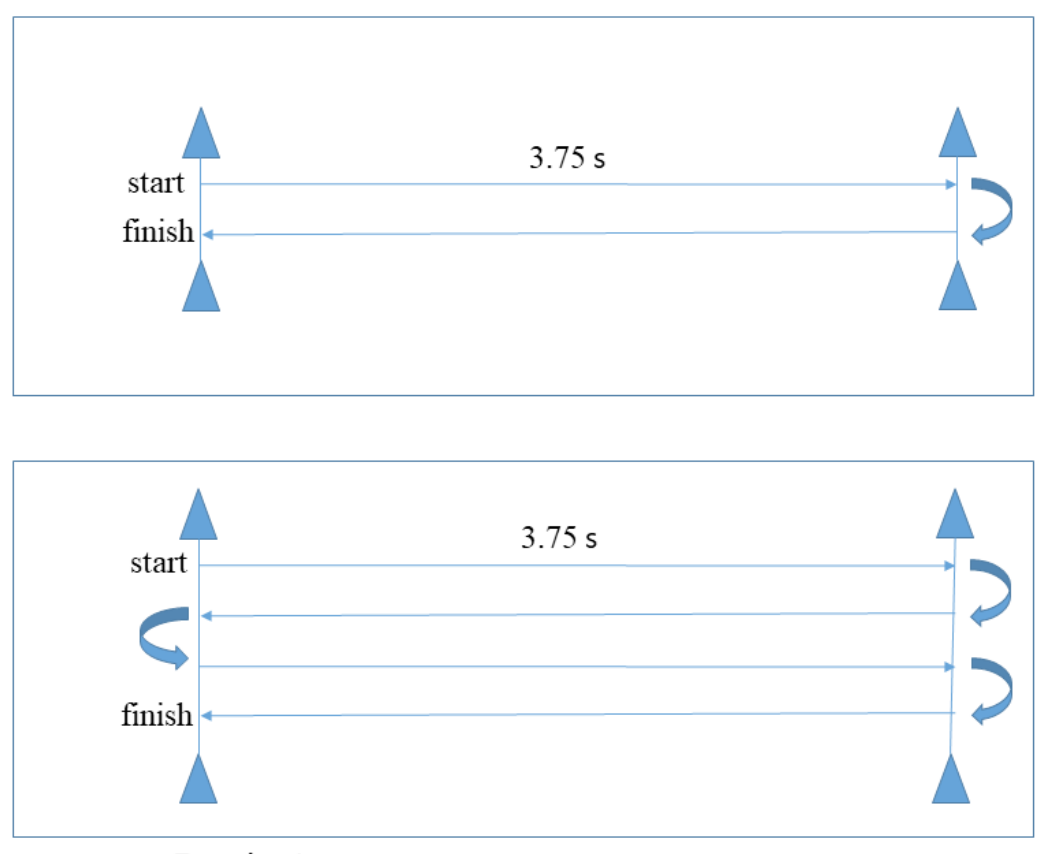

\begin{tabular}{|l} 
SRIT-3 \\
Number of sets: 4 x 4 min \\
Rest interval between sets: 3 min \\
Intensity: $86 \%$ PS $_{\mathrm{FIET}}$ \\
Number of bouts: 8 \\
Bout duration: $15 \mathrm{~s}$ \\
Rest interval between bouts: $15 \mathrm{~s}$ \\
Work-to-rest ratio: $1: 1$ \\
Number of directional changes: 3
\end{tabular}

\section{SRIT-1}

Number of sets: $4 \times 4 \mathrm{~min}$

Rest interval between sets: $3 \mathrm{~min}$

Intensity: $89 \% \mathrm{PS}_{\mathrm{FIET}}$

Number of bouts: 16

Bout duration: $7.5 \mathrm{~s}$

Rest interval between bouts: $7.5 \mathrm{~s}$

Work-to-rest ratio: 1:1

Number of directional changes: 1

\section{Change of Direction}

Figure 1. Schematic representation of the SRIT-1 (upper panel) and SRIT-3 (lower panel) training protocols implemented in the study

changes on [La], which was measured at the end of each set during the $1^{\text {st }}$ and the $10^{\text {th }}$ training session.

\section{Blood lactate responses}

Blood samples $(25 \mu \mathrm{l})$ were collected from the ear lobe into microcentrifuge tubes containing $50 \mu \mathrm{l} \mathrm{NaF}(1 \%)$ by a previously trained evaluator, and the [La] was determined with an electrochemical method (YSI 2700 STAT, YSI Incorporated, Yellow Springs, OH, USA) in our laboratory. Blood samples for [La] measurement were collected immediately after the end of the $1^{\text {st }}, 2^{\text {nd }}$, $3^{\text {rd }}$, and $4^{\text {th }}$ set during the $1^{\text {st }}$ and $10^{\text {th }}$ training sessions.

\section{Futsal Intermittent Endurance Test}

The FIET consists of shuttle-run bouts of $45 \mathrm{~m}$ (i.e., $3 \times 15 \mathrm{~m}$ ) performed at progressive speeds until exhaustion [26]. The running speed was controlled by a beep emitted by prerecorded audio cues. In order to increase the running speed, the frequency in which beep was emitted was progressively reduced. Every $45 \mathrm{~m}$, the participants were allowed to actively rest for $10 \mathrm{~s}$. After each $8 \times 45$-m bout, the players passively rested for $30 \mathrm{~s}$ before continuing. The starting speed was set at $9 \mathrm{~km} \cdot \mathrm{h}^{-1}$ and speed increments during the first $9 \times 45-\mathrm{m}$ bouts were of $0.33 \mathrm{~km} \cdot \mathrm{h}^{-1}$. After $9 \times$
45 -m bouts, the increment was shifted to $0.20 \mathrm{~km} \cdot \mathrm{h}^{-1}$ every $45 \mathrm{~m}$ until exhaustion. The test was finished when a participant did not reach the front line in time with beeps for 2 successive repetitions (objective criteria). PS $_{\text {FIET }}$ was calculated from the distance of the last set completed by the athlete (i.e., $45 \mathrm{~m}$ ) divided by the time to complete the stage repetition. In the case of an incomplete set, $\mathrm{PS}_{\text {FIET }}$ was interpolated with the use of the equation:

$$
\mathrm{PS}_{\mathrm{FIET}}=(s+[(n s / 8) \times 1.6])
$$

where $s$ is the speed of the last fully completed stage and $n s$ is the number of repetitions completed in the partially completed stage. The PS reached at the end of the test by the athletes was reported as the performance criterion for the FIET (PS FIET $_{\text {) }}$.

\section{Performance tests}

All performance tests were carried out on two different days of the week immediately before the commencement and after the cessation of the training intervention period, and included: (1) unloaded vertical jumping tests (countermovement jump [CMJ] and SJ); (2) isokinetic assessments of the lower limbs (Wednesday); and (3) a 40-m shuttle-run sprint test 
A.S. Teixeira et al., Shuttle-run training and female futsal

(Friday). These physical fitness tests were performed in a laboratory and on an indoor futsal court. Each assessment took place at the same time of the day (2:00-4:00 PM) in order to ensure similar environmental conditions. All players were familiarized with the testing procedures (as part of their regular physical performance assessment) before commencing the study.

\section{Vertical jumping tests}

Vertical jumping height $(\mathrm{cm})$ was determined with both the SJ and CMJ. In the SJ, the players were required to remain in a static position with a $90^{\circ}$ knee flexion angle for $2 \mathrm{~s}$ before jumping, without any preparatory movements. In the CMJ, the participants were instructed to execute a downward movement followed by a complete extension of the legs and were free to determine the countermovement amplitude to avoid changes in jumping coordination. The SJ and CMJ were executed with the hands fixed on the hips. All jumps were performed on a contact platform (Quattro Jump, Kistler, Switzerland). The total of 3 attempts were allowed for each jump. Successive attempts of the same jump mode were interspersed with ca. 45-s intervals. The best $\mathrm{SJ}$ and $\mathrm{CMJ}$ attempts were retained for analysis.

\section{Isokinetic assessments}

Isokinetic peak torque for the knee extensors of each player's dominant leg ('kicking leg') for both concentric and eccentric muscle actions (i.e., positive and negative work, respectively) were measured with a calibrated isokinetic dynamometer (Biodex System 3, Shirley, NY, USA) at an angular velocity of $60^{\circ} \cdot \mathrm{s}^{-1}$. The players performed a 10-min cycling warm-up on a cycle ergometer (Lode, Groningen, The Netherlands) with minimal resistance (basket supported) at $60 \mathrm{rpm}$. The participant was then placed in a seated position adjusted to the manufacturer's guidelines in a standardized $85^{\circ}$ hip flexion from the anatomical position. The lever arm of the dynamometer was aligned with the lateral epicondyle of the knee and the force pad was placed approximately $3-5 \mathrm{~cm}$ superior to the medial malleolus with the ankle in a plantigrade position. The range of motion during testing was set at $70^{\circ}$, taking $0^{\circ}$ as reference for maximum knee extension. Cushioning was set with the use of hard deceleration (in accordance with the manufacturer's guidelines). At the beginning of each session, the participant was asked to relax the leg so that passive determination of the effects of gravity on the limb and lever arm could be accounted for. Each player performed one set of 5 continuous maximal repetitions. All subjects were encouraged to give maximal effort for each action through both visual feedback and strong verbal encouragement. For subsequent analysis, the peak torques of the knee extensors from the best repetitions in both concentric and eccentric muscular actions were retained and expressed in $\mathrm{N} \cdot \mathrm{m}^{-1}$.

\section{Single 40-m shuttle-sprint test}

All futsal players performed a two single 40-m shuttle-sprint test, starting from a standing position of $0.5 \mathrm{~m}$ behind the start line, and times were recorded electronically via photocells (Speed Test 4.0, Brazil). Before starting, the participants were instructed to run as fast as possible between two lines placed $20 \mathrm{~m}$ apart, with the start/finish line (and the photocells) positioned at the midpoint of the course. Each player sprinted $10 \mathrm{~m}$ from the start/finish line to the end of the course, turned $180^{\circ}$, sprinted $20 \mathrm{~m}$ to the other end of the course, turned $180^{\circ}$, and sprinted $10 \mathrm{~m}$ back through the start/finish line. The fastest time was considered for the analyses. Owing to the specificity of the modality, a shuttle running sprint protocol was prioritized instead of a short distance linear sprint test. This shuttle running sprint protocol, despite involving a total distance $(40 \mathrm{~m})$ not compatible with the futsal match demand, requires that athlete perform two changes of direction of $180^{\circ}$ and three acceleration events during the course of the test. This protocol is closer to the pattern of movement that occurs during the game, such as those seen in situations of offensive and defensive transition.

\section{Statistical analysis}

The data in the text, tables, and figures are presented as means \pm standard deviations $(S D)$ or $\pm 90 \%$ confidence interval (90\% CI). All data were first logtransformed to reduce bias arising from the non-uniformity error. The data were then analysed for practical significance with the use of magnitude-based inferences (MBI) [27]. We applied this qualitative approach because traditional statistical approaches often do not indicate the magnitude of an effect, which is typically more relevant to athletic performance than any statistically significant effect. To examine the effects of the intervention (SRIT-1 and SRIT-3) on physiological and physical performance outcomes, differences between groups (SRIT-1 vs. SRIT-3) and over time (pre-train- 
ing vs. post-training) for all dependent variables were calculated with an MBI approach. The smallest worthwhile change was calculated $(0.2 \times S D)$ and $90 \%$ CIs were determined. Quantitative chances of beneficial/ better or harmful/worse effects were assessed qualitatively as follows: $<1 \%$, almost certainly not; $1-5 \%$, very unlikely; 5-25\%, unlikely; 25-75\%, possibly; 7595\%, likely; 95-99\%, very likely; and > 99\%, almost certainly. If the chance of having beneficial/better or harmful/worse performances were both $>5 \%$, the true difference was assessed as unclear [27]. In addition, the standardized mean difference or Cohen's $d$ effect size $(E S)$ of changes in physical performance measures were calculated. Threshold values for Cohen's $d E S$ statistics were: $\leqslant 0.2$ (trivial), $>0.2($ small $),>0.5($ moderate), and $>0.8$ (large). All inference-based analyses were conducted with the use of a publicly available spreadsheet (http://www.sportsci.org/resource/stats/).

\section{Ethical approval}

The research related to human use has been complied with all the relevant national regulations and institutional policies, has followed the tenets of the Declaration of Helsinki, and has been approved by the local research Ethics Committee (protocol 251.245).

\section{Informed consent}

Informed consent has been obtained from all individuals included in this study. All players aged under 18 provided a written informed consent from a parent or legal guardian.

\section{Results}

\section{Blood lactate responses}

In the first set of the $1^{\text {st }}$ training session, SRIT-3 presented a likely (94/03/03\%; ES = 1.43 [90\% CI: 0.112.76]) higher [La] than the SRIT-1 training model. When the remaining sets were compared, differences between the SRIT-1 and SRIT-3 groups for [La] were rated as unclear (ES: 0.44-0.49) in both moments $\left(1^{\text {st }}\right.$ and $10^{\text {th }}$ training session) (Figure 2). In the $1^{\text {st }}$ training session, [La] presented a possibly to very likely progressive increase over the sets (set $1<\operatorname{set} 2<\operatorname{set} 3<\operatorname{set} 4$ ) in both training regimens, while in the $10^{\text {th }}$ training session, [La] remained unchanged over the sets (set $1=\operatorname{set} 2=$ set $3=$ set 4 ). With the exception the first set of SRIT-1 (unclear effect), [La] was very likely decreased in the $10^{\text {th }}$ training session compared with the $1^{\text {st }}$ training session during all the other sets in both SRIT-1 and SRIT-3 groups (Figure 2).

\section{Baseline}

Between-group differences at baseline for all physical performance outcomes were rated as unclear (trivial to small ES) (Table 1).

\section{Within-group changes}

Raw values for all dependent variables, relative changes, and qualitative outcomes derived from within-group analysis are presented in Table 1 . After the
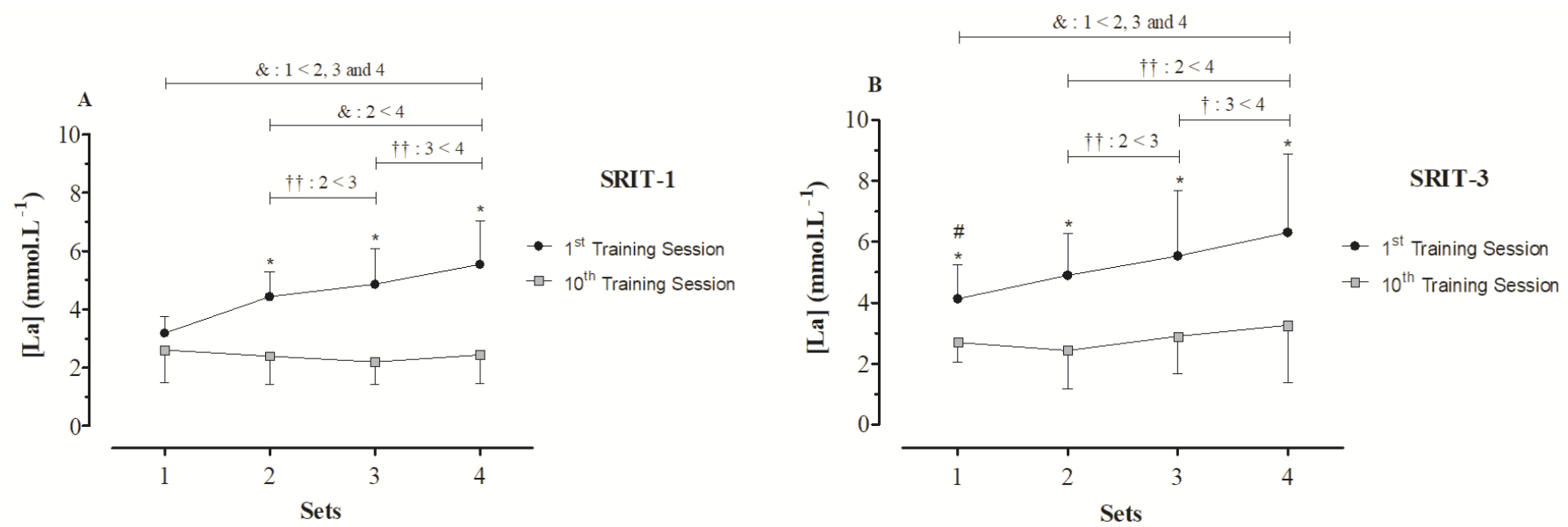

* very likely $\left(95-99 \%\right.$ chances that the true value of the statistic is practically meaningful) differences between the $1^{\text {st }}$ and the $10^{\text {th }}$ training session; \# a likely (75-95\% chances that the true value of the statistic is practically meaningful) difference between the SRIT-3 and SRIT-1 models in the first set of the $1^{\text {st }}$ training session; \& very likely differences between sets during the $1^{\text {st }}$ training session in both groups; †† likely difference between sets during the $1^{\text {st }}$ training session in both groups; $†$ possibly ( $25-75 \%$ chances that the true value of the statistic is practically meaningful) differences between sets during the $1^{\text {st }}$ training session in both groups

Figure 2. Blood lactate responses over the sets within both SRIT-1 (panel A) and SRIT-3 (panel B) training models during the $1^{\text {st }}$ and $10^{\text {th }}$ training sessions 


\section{HUMAN MOVEMENT}

A.S. Teixeira et al., Shuttle-run training and female futsal

Table 1. Descriptive statistics (mean \pm standard deviation) and changes (with $90 \%$ confidence limits) in physical performance measures following the two shuttle-run intermittent training (SRIT) modes (SRIT-1 and SRIT-3)

\begin{tabular}{cccccc}
\hline & Pre-training & Post-training & \multicolumn{3}{c}{ Magnitude-based inferences } \\
\hline $\begin{array}{c}\text { Training } \\
\text { model }\end{array}$ & Mean $\pm S D$ & Mean $\pm S D$ & $\%$ change & $E S$ & $\begin{array}{c}\text { Chances } \\
(\mathrm{B} / \mathrm{T} / \mathrm{H})\end{array}$
\end{tabular}

\begin{tabular}{|c|c|c|c|c|c|c|c|}
\hline $\begin{array}{l}40-1 \\
\text { spri }\end{array}$ & $\begin{array}{l}\text { SRIT-1 }(n=7) \\
\text { SRIT-3 }(n=7)\end{array}$ & $\begin{array}{l}4.52 \pm 0.13 \\
4.51 \pm 0.20\end{array}$ & $\begin{array}{l}4.57 \pm 0.16 \\
4.64 \pm 0.16\end{array}$ & $\begin{array}{c}1.0(-1.0 ; 3.1) \\
2.9(1.7 ; 4.1)\end{array}$ & $\begin{array}{l}0.33(-0.30 ; 0.95) \\
0.58(0.35 ; 0.80)\end{array}$ & $\begin{array}{l}64 / 28 / 08 \% \\
99 / 01 / 00 \%\end{array}$ & $\begin{array}{l}\text { Unclear } \\
\text { Very likely }\end{array}$ \\
\hline \multirow{2}{*}{$\mathrm{SJ}(\mathrm{cm})$} & & & & & & & \\
\hline & IT & & & 9.2 & & $98 / 02 / 00 \%$ & \\
\hline \multirow{2}{*}{$\mathrm{CMJ}(\mathrm{cm})$} & & & & & & & \\
\hline & SRIT- & & & 8.4 & & 98 & \\
\hline \multirow{2}{*}{$\operatorname{KEcon}\left(\mathrm{N} \cdot \mathrm{m}^{-1}\right)$} & & & & 4) & & & \\
\hline & SRIT-3 $(n=7)$ & $131.7 \pm 22.4$ & $154.4 \pm$ & $17.3^{3}$ & 0.88 & $96 / 03 / 01 \%$ & Very likel \\
\hline \multirow{2}{*}{$\operatorname{KEecc}\left(\mathrm{N} \cdot \mathrm{m}^{-1}\right)$} & RIT-1 & $263.6 \pm 30.4$ & $74.0 \pm 30.4$ & $6.3 ; 14.1)$ & 0.30 & $60 / 29 / 11 \%$ & thai \\
\hline & SRIT-3 $(n=7)$ & $247.7 \pm 34.0$ & $271.9 \pm 42.6$ & $7 ; 14.4)$ & $.30 ; 0.94)$ & $98 / 02 / 00 \%$ & Very likel \\
\hline
\end{tabular}

SJ - squat jump, CMJ - countermovement jump, KEcon - knee extensors concentric peak torque,

KEecc - knee extensors eccentric peak torque, B - chance of a beneficial change, $\mathrm{T}$ - trivial change,

$\mathrm{H}$ - chance of a harmful change

Table 2. Changes observed for the shuttle-run intermittent training (SRIT): SRIT-3 compared with SRIT-1

\begin{tabular}{lccccc}
\cline { 2 - 5 } & \% change $(90 \% \mathrm{CI})$ & ES $(90 \% \mathrm{CI})$ & Rating & Chances $(\mathrm{B} / \mathrm{T} / \mathrm{H})$ & Outcomes \\
\hline $40-\mathrm{m}$ shuttle sprint $\left(\mathrm{m} \cdot \mathrm{s}^{-1}\right)$ & $1.8(-0.4 ; 4.1)$ & $0.48(-0.10 ; 1.07)$ & Small & $80 / 17 / 03 \%$ & Likely \\
SJ $(\mathrm{cm})$ & $1.3(-6.0 ; 9.2)$ & $0.11(-0.71 ; 0.93)$ & Trivial & $43 / 32 / 25 \%$ & Unclear \\
CMJ $(\mathrm{cm})$ & $1.3(-5.2 ; 8.2)$ & $0.09(-0.68 ; 0.86)$ & Trivial & $40 / 34 / 26 \%$ & Unclear \\
KEcon $\left(\mathrm{N} \cdot \mathrm{m}^{-1}\right)$ & $-0.8(-16.7 ; 18.0)$ & $-0.04(-0.76 ; 0.68)$ & Trivial & $28 / 37 / 35 \%$ & Unclear \\
KEecc $\left(\mathrm{N} \cdot \mathrm{m}^{-1}\right)$ & $5.9(-4.5 ; 17.4)$ & $0.41(-0.38 ; 1.19)$ & Small & $68 / 22 / 10 \%$ & Unclear \\
\hline
\end{tabular}

SJ - squat jump, CMJ - countermovement jump, KEcon - knee extensors concentric peak torque,

KEecc - knee extensors eccentric peak torque, $E S$ - effect size, B - chance of a beneficial change, T - trivial change,

$\mathrm{H}$ - chance of a harmful change

SRIT-3 training, very likely moderate improvements occurred in the 40-m sprint (2.9\%; 90\% CI: 1.7-4.1), while changes were unclear following the SRIT-1 training. Changes in the SJ and CMJ height were likely (large ES) and very likely (moderate ES) beneficial following the SRIT-1 and SRIT-3 training protocols, respectively. The knee extensors concentric peak torque was likely (89/10/01\%) and very likely (96/03/01\%) increased at post-training period for the SRIT-1 and SRIT-3 groups, respectively. The knee extensors eccentric peak torque was very likely improved after the SRIT-3 training regimen, but unclear following SRIT-1.

\section{Between-group differences}

The results of the between-group analysis are presented in Table 2 and illustrated in Figure 3. Following the SRIT-3 training, changes in the 40-m shuttle- sprint speed were likely $(80 / 17 / 03 \% ; E S=0.48$ [90\% CI: from -0.10 to 1.07$])$ greater than those observed in SRIT-1. Between-groups differences in the changes in the SJ and CMJ height, as well as knee extensors concentric and eccentric peak torque were unclear (trivial to small $E S)$.

\section{Discussion}

The original finding of the present study is that both SRIT-1 and SRIT-3 training models were effective at improving endurance capacity (inferred by [La] values during the same intermittent training mode), isokinetic strength, and jumping abilities, but only the SRIT-3 model improved sprinting speed (i.e., 40-m shuttle sprint) in female futsal players during a sprint test involving change of direction. The SRIT-3 training regimen (with more directional changes) induced a likely 
(80/17/03\%) superior gain in the 40-m shuttle sprint compared with SRIT-1 (Figure 3). This confirms our initial hypothesis that futsal-specific shuttle-run training approaches with more directional changes, probably because of the higher number of horizontal accelerations, might be more effective than models with fewer changes of direction to induce greater adaptations in the 40-m shuttle sprint performance among female futsal players.

The acute effect of intermittent running exercises with changes of direction on physiological, perceptual, and neuromuscular responses in team-sport athletes has been extensively addressed [16, 17, 28, 29]. To a lesser extent, longitudinal training interventions (4-6-week) have investigated the effects of distinct shuttle-run training models (maximal and all-out efforts) on athletic performance in soccer and basketball players [30, 31]. In theory, training models involving shuttle-run exercises would be expected to induce superior gains in performance owing to their higher acute metabolic, cardiorespiratory, neuromuscular, and perceptual responses [16, 17, 28, 29, 32]. In fact, a recent study involving young female basketball players showed that increasing the number of changes of direction during SRIT induced larger gains in agility, repeated-sprint ability, and intermittent endurance performance [20]. On the other hand, no superior adaptation in performance was reported in two recent works conducted by Da Silva et al. [30] and Attene et al. [31]. In accordance with Sanchez-Sanchez et al. [20] and contrary to the results obtained by Da Silva et al. [30] and Attene et al. [31], our findings show that if physical conditioning professionals implement the SRIT-3 training model in futsal female players, it could result in superior gains in the 40-m shuttle-sprint speed. The sample characteristics (female vs. male), sport modality (futsal vs. soccer/basketball), and training intensities (submaximal vs. maximal/all-out) differed between the current investigation and prior studies $[30,31]$ and this can serve as a basis to explain the controversial findings.

From the metabolic point of view, both shuttle-run protocols used in our study based on the rate of blood lactate accumulation in the $1^{\text {st }}$ training session (4.87 \pm 1.88 and $6.38 \pm 3.20 \mathrm{mmol} / \mathrm{l} / 5 \mathrm{~min}$ for SRIT- 1 and SRIT-3, respectively) can be, at least, categorized as mildly anaerobic (> $4 \mathrm{mmol} / \mathrm{l} / 5 \mathrm{~min}$ ) according to the classification proposed by Buchheit and Laursen [33]. Our training models induced low to moderate [La] values at the end of each set (ranging from 2.73 to $6.31 \mathrm{mmol} / \mathrm{l}$ ) [33]. Training-induced changes on physiological markers (e.g. blood [La], HR, and HR recovery at a given submaximal running intensity) are among the most common measures used to monitor metabolic and cardiovascular adaptations after a chronic exposure to interval training programs in team-sport

\section{SRIT-3 compared to SRIT-1}

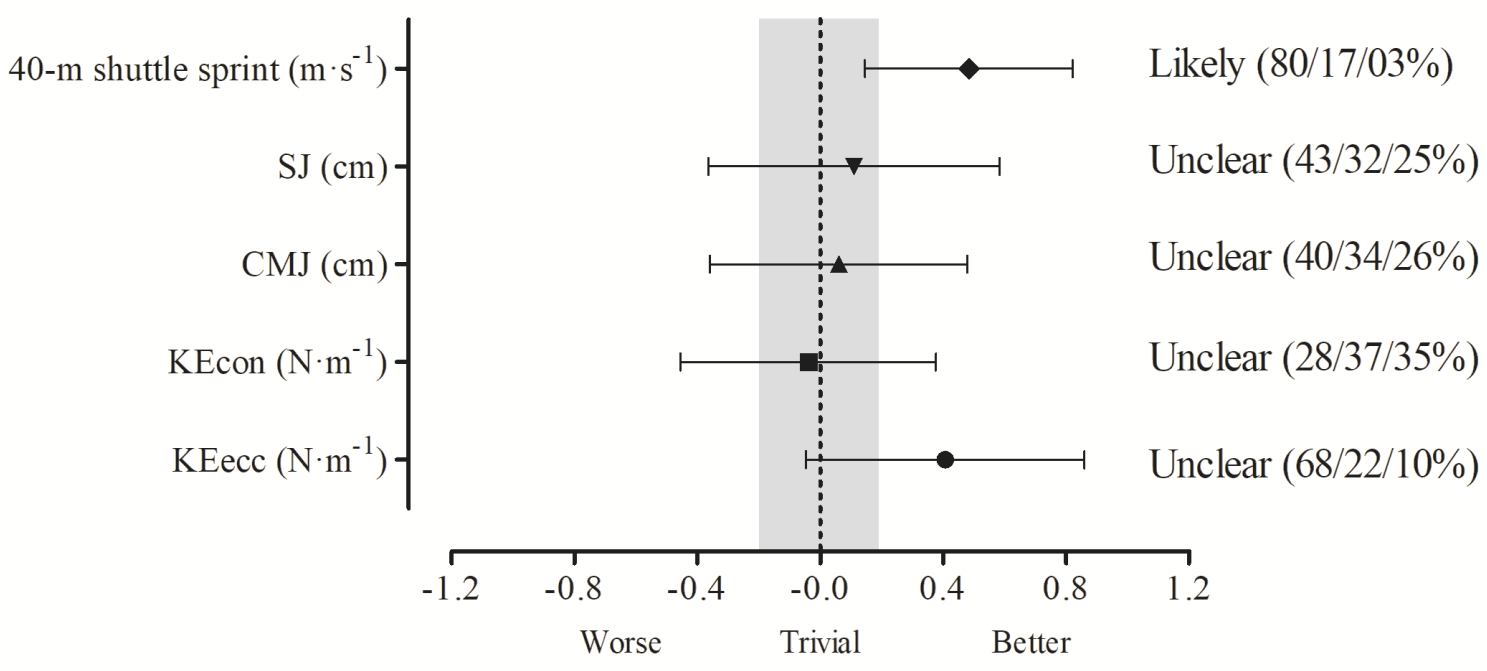

Standardized difference

SRIT - shuttle-run intermittent training, SJ - squat jump, CMJ - countermovement jump, KEcon - knee extensors concentric peak torque, KEecc - knee extensors eccentric peak torque

Figure 3. Effectiveness of the SRIT-3 compared with SRIT-1 training to improve 40-m shuttle sprint, SJ, CMJ, and isokinetic strength of the lower limbs. The grey area represents the smallest worthwhile change $(0.20)$

based on Cohen's principles 
A.S. Teixeira et al., Shuttle-run training and female futsal

players [34]. In the present study, [La] values in the SRIT-1 and SRIT-3 models were very likely decreased from the $1^{\text {st }}$ to $10^{\text {th }}$ training session (running at the same absolute intensity in the FIET) (Figure 2). This reduction in [La] is a classical training-induced adaptation in athletes [23]. The reduction in [La] for the same absolute exercise intensity following SRIT may result from a decrease in the rate of lactate production (possibly because of a lower rate of muscle glycogen utilization or a faster oxygen uptake kinetics that may increase the initial $\mathrm{O}_{2}$ availability/utilization) or from an increase in the ability to exchange and remove lactate from the blood [23]. Furthermore, this specific metabolic adaptation points at functional improvements in the endurance capacity of the athletes [23]. In practical terms, this increased aerobic fitness of the players suggests that they will possibly cope better (i.e., increased tolerance) with the high workloads of specific training sessions and competitions [35, 36].

The effectiveness of isolated strength or plyometric and combined strength-plyometric training to enhance key aerobic fitness markers has received attention [37]. However, the current understanding about the effects of futsal-specific shuttle-run training regimen on strength-power and sprinting speed adaptations in team-sport athletes are less clear when compared with other training strategies, such as plyometrics. Controversial findings have been reported, with studies showing either improved [38] or unchanged [39-41] single sprint and vertical jump performances following aerobic interval training interventions. Traditionally, aerobic interval training programs are not primarily designed to optimize power-speed-related capacities in team-sport athletes, but rather to induce positive cardiopulmonary and metabolic adaptations. Interestingly, our findings showed that SJ and CMJ heights were likely to very likely increased from pre- to posttraining periods in both SRIT-1 and SRIT-3 groups. These results are in accordance with McMillan et al. [38], who observed a higher jumping ability after an interval training model in male soccer players. The relative changes in $\mathrm{SJ}$ and $\mathrm{CMJ}$ performances in our training model (7-8\%) were comparable to the gains (6.5-10.0\%) demonstrated in amateur female soccer players after low-volume plyometric training sessions $[15,42]$. The improvements reported here are also similar to those described in a recent meta-analysis, showing that plyometric training induces moderate to large increases in jumping ability in female athletes [43]. It is reasonable to assume that the mechanical load generated by intermittent shuttle run, such as the SRIT-1 and SRIT-3 models, is probably superior to constant speed running [16, 28, 32]. The greater mechanical requirement to constantly change direction in shuttle-run exercises may have elicited significant adaptations in motor unit synchronization, stretch-shortening cycle efficiency, or musculotendinous stiffness, which are considered critical elements of jump performance [44]. Thus, our data suggest that SRIT models with an increased number of direction changes could be incorporated alone or (preferentially) along with certain training strategies (e.g. plyometrics) for improving jumping ability, particularly in female futsal players. However, the extrapolation of these results to male team-sport athletes should be made with caution and still deserves further investigations.

Sprint running acceleration is also a key feature of physical performance in team sports (e.g. soccer, futsal) [16, 28]. Accelerations, even at submaximal speeds, are also physiologically and mechanically relevant for athletes from different sports disciplines [16]. A practical strategy to increase the number of accelerations during shuttle intermittent running exercises is to manipulate the frequency of changes of direction [16, 28]. This manipulation will impact on the number of times that players should apply force horizontally into the ground to overcome the inertia and move their bodies forward as rapidly as possible throughout a given workout. Recent studies have shown that the development of training strategies (e.g., horizontally-directed exercises) which prioritize the application of force in the horizontal direction are paramount to significantly improve sprinting speed [45-47]. The present study found that the $40-\mathrm{m}$ shuttle-sprint speed was very likely (99\% chances of a real effect) improved after SRIT-3, but unclear changes $(64 / 28 / 08 \%$; ES $=0.33)$ were noticed following the SRIT-1 training model. Thus, SRIT-3 was likely (80/17/035) more effective than SRIT-1 for improving shuttle sprinting speed in female futsal players. This superior gain on 40-m shuttlesprint speed might be attributed to specific adaptations on muscle mechanical properties, as a result of the higher time spent accelerating per running bout in the SRIT-3 model, which also implies in longer periods of time applying horizontal forces. Dello Iacono et al. [45] also found that horizontal-drop jump training resulted in larger gains in short-sprint (i.e., 10-m) and change of direction capabilities in comparison with the vertical-drop jump group. Additionally, McKenzie et al. [48] showed that horizontal jump performance significantly improved after individualized optimal handheld loading in comparison with unloaded jumps (i.e., control) in female netball players. This observation was attributed to the increased eccen- 
tric activity and greater technical ability to produce horizontal forces in the loaded jump condition. As sprints and their repetition during games are key aspects of futsal competition [2], being usually related to goals scored and competitive success in team-sports [49], the greater adaptations in sprinting speed reported with SRIT-3 could be considered an important advantage of this specific training strategy.

Some methodological limitations should also be noted. First, it was not possible to determine the impact of the training on technical-tactical variables (e.g., number of involvement with the ball) in the current investigation. Second, the lack of a control group does not allow to ensure that positive changes on [La] values were solely elicited by the applied training, without any influence of other factors. However, the use of controls is difficult when dealing with elite female futsal players.

\section{Practical applications}

Owing to the congested calendars and short-duration preseasons in elite team sports, strength and conditioning coaches are required to implement training sessions which are capable of optimizing multiple fitness components concomitantly. This study showed that combining high-intensity interval runs with changes of direction could be an alternative to counteract the previously reported interference phenomenon in futsal players. In other words, this training mode is able to improve aerobic endurance concomitantly with isokinetic strength, jumping ability, and performance in a 40-m shuttle-sprint test. Since SRIT-3 was more effective than SRIT-1 in the test, we strongly advise futsal professionals to include a high volume of change of directions during supramaximal interval runs to stress the braking force and horizontal propulsive force during each manoeuvre in order to elicit neuromuscular adaptations that are considered essential in this sport.

\section{Acknowledgments}

The authors wish to acknowledge the committed participation of all female futsal players, the support staff, and coaches involved in this study.

\section{Funding}

This research was financially supported by the Brazilian National Council for Scientific and Technological Development (CNPq, Conselho Nacional de Desenvolvimento Científico e Tecnológico).

\section{Disclosure statement}

No author has any financial interest or received any financial benefit from this research.

\section{Conflict of interest}

The authors state no conflict of interest.

\section{References}

1. Beato M, Coratella G, Schena F. Brief review of the state of art in futsal. J Sports Med Phys Fitness. 2016;56(4): 428-432.

2. Caetano FG, de Oliveira MJ, Marche AL, Nakamura FY, Cunha SA, Moura FA. Characterization of the sprint and repeated-sprint sequences performed by professional futsal players, according to playing position, during official matches. J Appl Biomech. 2015;31(6):423429; doi: 10.1123/jab.2014-0159.

3. Castagna C, D’Ottavio S, Granda Vera J, Barbero Alvarez JC. Match demands of professional futsal: a case study. J Sci Med Sport. 2009;12(4):490-494; doi: 10.1016/j.jsams.2008.02.001.

4. Barbero-Alvarez J, Soto VM, Barbero-Alvarez V, Granda-Vera J. Match analysis and heart rate of futsal players during competition. J Sports Sci. 2008;26(1):6373; doi: 10.1080/02640410701287289.

5. De Oliveira Bueno MJ, Caetano FG, Pereira TJ, De Souza NM, Moreira GD, Nakamura FY, et al. Analysis of the distance covered by Brazilian professional futsal players during official matches. Sport Biomech. 2014; 13(3):230-240; doi: 10.1080/14763141.2014.958872.

6. Oliveira RS, Leicht AS, Bishop D, Barbero-Álvarez JC, Nakamura FY. Seasonal changes in physical performance and heart rate variability in high level futsal players. Int J Sports Med. 2013;34(5):424-430; doi: 10.1055/s-0032-1323720.

7. De Freitas VH, Pereira LA, de Souza EA, Leicht AS, Bertollo M, Nakamura FY. Sensitivity of the Yo-Yo Intermittent Recovery Test and cardiac autonomic responses to training in futsal players. Int J Sports Physiol Perform. 2015;10(5):553-558; doi: 10.1123/ ijspp.2014-0365.

8. Pedro RE, Milanez VF, Boullosa DA, Nakamura FY. Running speeds at ventilatory threshold and maximal oxygen consumption discriminate futsal competitive level. J Strength Cond Res. 2013;27(2):514-518; doi: 10.1519/JSC.0b013e3182542661.

9. Nakamura FY, Pereira LA, Rabelo FN, Ramirez-Campillo R, Loturco I. Faster futsal players perceive higher training loads and present greater decreases in sprinting speed during the preseason. J Strength Cond Res. 2016;30(6):1553-1562; doi: 10.1519/JSC.0000000000 001257.

10. Yanci J, Castillo D, Iturricastillo A, Ayarra R, Nakamura FY. Effects of two different volume-equated weekly distributed short-term plyometric training programs on futsal players' physical performance. J Strength Cond 
A.S. Teixeira et al., Shuttle-run training and female futsal

Res. 2017;31(7):1787-1794; doi: 10.1519/JSC.000000 0000001644.

11. Fyfe JJ, Bishop DJ, Stepto NK. Interference between concurrent resistance and endurance exercise: molecular bases and the role of individual training variables. Sports Med. 2014;44(6):743-762; doi: 10.1007/s40279 -014-0162-1.

12. Loturco I, Pereira L, Kobal R, Zanetti V, Gil S, Kitamura K, et al. Half-squat or jump squat training under optimum power load conditions to counteract power and speed decrements in Brazilian elite soccer players during the preseason. J Sports Sci. 2015;33(12):12831292; doi: 10.1080/02640414.2015.1022574.

13. Miloski B, de Freitas VH, Nakamura FY, de A. Nogueira FC, Bara-Filho MG. Seasonal training load distribution of professional futsal players: effects on physical fitness, muscle damage and hormonal status. J Strength Cond Res. 2016;30(6):1525-1533; doi: 10.1519/JSC. 0000000000001270.

14. Docherty D, Sporer B. A proposed model for examining the interference phenomenon between concurrent aerobic and strength training. Sports Med. 2000;30(6): 385-394; doi: 10.2165/00007256-200030060-00001.

15. Ramírez-Campillo R, Vergara-Pedreros M, HenríquezOlguín C, Martínez-Salazar C, Alvarez C, Nakamura FY, et al. Effects of plyometric training on maximal-intensity exercise and endurance in male and female soccer players. J Sports Sci. 2015;34(8):687-693; doi: 10.1080/02640414.2015.1068439.

16. Akenhead R, French D, Thompson KG, Hayes PR. The physiological consequences of acceleration during shuttle running. Int J Sports Med. 2015;36(4):302-307; doi: 10.1055/s-0034-1389968.

17. Dellal A, Keller D, Carling C, Chaouachi A, Wong DP, Chamari K. Physiologic effects of directional changes in intermittent exercise in soccer players. J Strength Cond Res. 2010;24(12):3219-3226; doi: 10.1519/JSC. 0b013e3181b94a63.

18. Buchheit M. The 30-15 intermittent fitness test: accuracy for individualizing interval training of young intermittent sport players. J Strength Cond Res. 2008;22(2): 365-374; doi: 10.1519/JSC.0b013e3181635b2e.

19. Buchheit M, Laursen PB. High-intensity interval training, solutions to the programming puzzle: Part I: cardiopulmonary emphasis. Sports Med. 2013;43(5):313338; doi: 10.1007/s40279-013-0029-x.

20. Sanchez-Sanchez J, Carretero M, Ramirez-Campillo R, Petisco C, Diego M, Gonzalo-Skok O, et al. Effects of high-intensity training with one versus three changes of direction on youth female basketball players' performance. Kinesiology. 2018;50(Suppl. 1):117-125.

21. Barbero-Alvarez JC, Subiela JV, Granda-Vera J, Castagna C, Gómez M, Del Coso J. Aerobic fitness and performance in elite female futsal players. Biol Sport. 2015; 32(4):339-344; doi: 10.5604/20831862.1189200.

22. Loturco I, Pereira LA, Kobal R, Zanetti V, Kitamura K, Abad CC, et al. Transference effect of vertical and hori- zontal plyometrics on sprint performance of high-level U-20 soccer players. J Sports Sci. 2015;33(20):21822191; doi: 10.1080/02640414.2015.1081394.

23. Jones AM, Carter H. The effect of endurance training on parameters of aerobic fitness. Sports Med. 2000;29(6): 373-386; doi: 10.2165/00007256-200029060-00001.

24. Soares-Caldeira LF, de Souza EA, de Freitas VH, de Moraes SM, Leicht AS, Nakamura FY. Effects of additional repeated sprint training during preseason on performance, heart rate variability, and stress symptoms in futsal players: a randomized controlled trial. J Strength Cond Res. 2014;28(1):2815-2826; doi: 10.1519/JSC.0000000000000461.

25. Guglielmo LGA, Arins FB, De Lucas RD, Carminatti LJ. The effects of five weeks of high-intensity interval training on physiological indices in female futsal players: a comparison between two models. $20^{\text {th }}$ Annual Congress of the European College of Sport Science; 2015; 602-603.

26. Castagna C, Barbero Alvarez JC. Physiological demands of an intermittent futsal-oriented high-intensity test. J Strength Cond Res. 2010;24(9):2322-2329; doi: 10.1519/JSC.0b013e3181e347b9.

27. Hopkins WG, Marshall SW, Batterham AM, Hanin J. Progressive statistics for studies in sports medicine and exercise science. Med Sci Sports Exerc. 2009;41(1):313; doi: 10.1249/MSS.0b013e31818cb278.

28. Fessi MS, Farhat F, Dellal A, Malone JJ, Moalla W. Straight-line and change-of-direction intermittent running in professional soccer players. Int J Sports Physiol Perform. 2018;13(5):562-567; doi: 10.1123/ ijspp.2016-0318.

29. Buchheit M, Bishop D, Haydar B, Nakamura FY, Ahmaidi S. Physiological responses to shuttle repeatedsprint running. Int J Sports Med. 2010;31(6):402-409; doi: 10.1055/s-0030-1249620.

30. Da Silva JF, Nakamura FY, Carminatti LJ, Dittrich N, Cetolin T, Guglielmo LG. The effect of two generic aerobic interval training methods on laboratory and field test performance in soccer players. J Strength Cond Res. 2015;29(6):1666-1672; doi: 10.1519/JSC.000000000 0000776.

31. Attene G, Nikolaidis PT, Bragazzi NL, Dello Iacono A, Pizzolato F, Zagatto AM, et al. Repeated sprint ability in young basketball players (part 2): the chronic effects of multidirection and of one change of direction are comparable in terms of physiological and performance responses. Front Physiol. 2016;7:262; doi: 10.3389/ fphys.2016.00262.

32. Hader K, Mendez-Villanueva A, Ahmaidi S, Williams BK, Buchheit M. Changes of direction during high-intensity intermittent runs: neuromuscular and metabolic responses. BMC Sports Sci Med Rehabil. 2014;6:2; doi: 10.1186/2052-1847-6-2.

33. Buchheit M, Laursen PB. High-intensity interval training, solutions to the programming puzzle. Part II: anaerobic energy, neuromuscular load and practical ap- 
plications. Sports Med. 2013;43(10):927-954; doi: 10.1007/s40279-013-0066-5.

34. Buchheit M, Simpson MB, Al Haddad H, Bourdon PC, Mendez-Villanueva A. Monitoring changes in physical performance with heart rate measures in young soccer players. Eur J Appl Physiol. 2012;112(2):711-723; doi: 10.1007/s00421-011-2014-0.

35. Milanez VF, Pedro RE, Moreira A, Boullosa DA, SalleNeto F, Nakamura FY. The role of aerobic fitness on session rating of perceived exertion in futsal players. Int $\mathrm{J}$ Sports Physiol Perform. 2011;6(3):358-366; doi: 10.1123/ijspp.6.3.358.

36. Miloski B, Moreira A, Andrade FC, Freitas VH, Peçanha T, Nogueira RA, et al. Do physical fitness measures influence internal training load responses in high-level futsal players? J Sports Med Phys Fitness. 2014;54(5): 588-594.

37. Denadai BS, de Aguiar RA, de Lima LC, Greco CC, Caputo F. Explosive training and heavy weight training are effective for improving running economy in endurance athletes: a systematic review and meta-analysis. Sports Med. 2017;47(3):545-554; doi: 10.1007/ s40279-016-0604-z.

38. McMillan K, Helgerud J, Macdonald R, Hoff J. Physiological adaptations to soccer specific endurance training in professional youth soccer players. Br J Sports Med. 2005;39(5):273-277; doi: 10.1136/bjsm.2004. 012526 .

39. Helgerud J, Engen LC, Wisloff U, Hoff J. Aerobic endurance training improves soccer performance. Med Sci Sports Exerc. 2001;33(11):1925-1931; doi: 10.1097/ 00005768-200111000-00019.

40. Buchheit M, Laursen PB, Kuhnle J, Ruch D, Renaud C, Ahmaidi S. Game-based training in young elite handball players. Int J Sports Med. 2009;30(4):251-258; doi: 10.1055/s-0028-1105943.

41. Ferrari Bravo D, Impellizzeri FM, Rampinini E, Castagna C, Bishop D, Wisloff U. Sprint vs. interval training in football. Int J Sports Med. 2008;29(8):668674; doi: 10.1055/s-2007-989371.

42. Ramírez-Campillo R, González-Jurado JA, Martínez C, Nakamura FY, Peñailillo L, Meylan CM, et al. Effects of plyometric training and creatine supplementation on maximal-intensity exercise and endurance in female soccer players. J Sci Med Sport. 2016;19(8): 682-687; doi: 10.1016/j.jsams.2015.10.005.

43. Stojanović E, Ristić V, McMaster DT, Milanović Z. Effect of plyometric training on vertical jump performance in female athletes: a systematic review and meta-analysis. Sports Med. 2017;47(5):975-986; doi: 10.1007/ s40279-016-0634-6.

44. Markovic G, Mikulic P. Neuro-musculoskeletal and performance adaptations to lower-extremity plyometric training. Sports Med. 2010;40(10):859-895; doi: 10.2165/11318370-000000000-00000.

45. Dello Iacono A, Martone D, Milic M, Padulo J. Verticalvs. horizontal-oriented drop jump training: chronic effects on explosive performances of elite handball players. J Strength Cond Res. 2017;31(4):921-931; doi: 10.1519/JSC.0000000000001555.

46. Morin JB, Petrakos G, Jiménez-Reyes P, Brown SR, Samozino P, Cross MR. Very-heavy sled training for improving horizontal-force output in soccer players. Int J Sports Physiol Perform. 2017;12(6):840-844; doi: 10.1123/ijspp.2016-0444.

47. Loturco I, Kobal R, Kitamura K, Cal Abad CC, Faust B, Almeida L, et al. Mixed training methods: effects of combining resisted sprints or plyometrics with optimum power loads on sprint and agility performance in professional soccer players. Front Physiol. 2017;8:1034; doi: 10.3389/fphys.2017.01034.

48. McKenzie CR, Brughelli M, Whatman C, Brown SR. The influence of optimal handheld load on the technical ability to apply ground reaction forces during horizontal jumping in female netball players. Int J Sports Med. 2016;37(4):318-323; doi: 10.1055/s-0035 $-1565052$.

49. Faude O, Koch T, Meyer T. Straight sprinting is the most frequent action in goal situations in professional football. J Sports Sci. 2012;30(7):625-631; doi: 10.1080/ 02640414.2012 .665940 . 